\title{
Dos casos de evaluación intergubernamental en Canadá: El ajuego paralelo" y la cooperación sin consecuencias en las políticas públicas
}

Robert V. Segsworth * y Dale H. Poel **

Este artículo presenta una breve panorámica de los temas actuales en el federalismo canadiense y considera el papel de la evaluación formal en el contexto del federalismo y el sistema parlamentario de Canadá. Se presentan dos casos de evaluación intergubernamental para subrayar la implementación y el uso de programas de Evaluación en este contexto. El primero es una Evaluación sobre el Programa de oportunidades de empleo iniciado por el Ministerio de la Comunidad y Asuntos Sociales de Ontario. El segundo es la evaluacion del Plan de Asistencia Jurídica en Ontario iniciado conjuntamente por el Departamento Federal de Justicia y el Ministerio del Justicia de Ontario.

\section{Conflictos de sistemas y políticas públicas \\ en el federalismo canadiense}

Una lectura de la Constitución de Canadá daría a cualquier persona la impresion de que Canadá es una especie de Estado federal "clásico" a modo del descrito en el influyente libro de K.C. WHEARE, Federal Government (1946)

El artículo 91 atribuye la competencia exclusiva al Gobierno Federal para un amplio espectro de materias.

El articulo 92 dispone lo mismo para los gobiernos provinciales.

Otros artículos señalan áreas de competencias concurrentes e indican qué nivel de gobierno tiene preferencia en caso de conflicto de politicas federales y provinciales sobre una misma materia.

Existe una cláusula residual que atribuye la competencia al Gobierno Federal para todo aquello que no esté expresamente atribuido a los gobiernos provinciales.

La Constitución indica que el Gobierno Federal establecerá con total libertad el sistema tributario, no así los gobiernos pro- vinciales cuyo sistema impositivo queda restringido a los esquemas de la imposición directa.

Un cuidadoso estudio de las políticas federales canadienses nos hace dudar de la aparente claridad de los dos sistemas competenciales coeexistentes en un Estado federal.

En las últimas décadas del federalismo canadiense se aprecia el conflicto, la cooperación, la competición y una gradual descentralización del poder del gobierno nacional en Otawa hacia las capitales de las provincias.

\section{Más de dos niveles de gobierno}

Aunque constitucionalmente en Canadá existen sólo dos niveles de gobierno, se pueden fácilmente encontrar, al menos, otros dos niveles más. En la provincia de Ontario, por ejemplo, el Área Metropolitana de Toronto es una forma de gobierno regional (municipalidad regional) y la ciudad de Etobicoke representa el clásico gobierno local (referido, a veces, como un área de municipalidad). Estos dos niveles de gobierno son "criaturas" del gobierno provincial, pero pueden ser políticamente muy poderosos.

Por ejemplo, la provincia "Prince Eduard Island" elige sólo cuatro miembros de la Cámara de los Comunes, mientras que el Área Metropolitana de Toronto está representada por aproximadamente treinta y cinco Miembros en el Parlamento.

Además, para algunos propósitos, los grupos de provincias se reflejan de distintas maneras.

Por ejemplo, en la política de desarrollo económico, el Gobierno Federal ha creado la Agencia de Oportunidades del Canadá Atlántico (ACOA) para la asistencia de las cuatro provincias orientales de Newfoundland, Prince Edward Island, Nova Scotia y New Birunswick. Los gobernadores de las tres provincias marítimas se reunen regularmente en la "Conferencia de Gober- 
nadores Marítimos" para discutir los problemas comunes y desarrollar respuestas colectivas. Recientemente el gobernador de Newfoundland se ha incorporado a este grupo. En el mismo sentido, el Departamento para la diversificacion económica del Oeste (W.E.D) es la unidad del Gobierno Federal encargada de implementar la política de desarrollo regional en las cuatro provincias Occidentales de Manitoba, Saskatchewan, Alberta y British Columbia.

Los gobernadores occidentales también se reunen regularmente para alcanzar los mismos objetivos de sus homólogos del Canadá Atlántico.

Sin embargo, en algunos casos, el área geográfica afectada por las políticas y estructuras federales es de ámbito sub-provincial. Un ejemplo clásico es el FEDNOR, agencia federal encargada de la implementación de las políticas de desarrollo económico federal en la parte norte de la provincia de Ontario. En otras ocasiones, las iniciativas de politicas públicas pueden involucrar a todos los niveles de Gobierno del país.

Poco después de ser elevado al poder en 1993, el Gobierno Federal Liberal anunció un programa de infraestructuras de seis billones de dólares. Esto era un esfuerzo para estimular la economía y reducir la alta tasa de desempleo. El nuevo programa requeria la aportación de dos billones de dólares por parte del Gobierno Federal, otros dos billones provenientes de los gobiernos provinciales $y$, por último, los gobiernos municipales y regionales contribuyeron con otros dos billones.

Aunque la Constitución establece sólo dos niveles de gobierno, la realidad es que los gobiernos locales en sus distintas modalidades tienen una influencia significativa y frecuente, en las políticas y programas de los gobiernos federal y provinciales.

También debemos reseñar que la práctica del federalismo es algo dinámico y cambiante. Según el artículo de MALLORY (1965) existen "cinco caras" del federalismo que van desde un Estado cuasi-unitario hasta una federación crecientemente descentralizada con algunas fases regresivas en la tendencia descentralizadora. Desde la segunda guerra mundial, la tendencia descentralizadora del poder Federal al provincial se ha ido consolidando. Según ha avanzado el proceso descentralizador, se ha producido un cambio del federalismo clásico, al federalismo cooperativo $y$, por fin, al federalismo ejecutivo SMILEY (1987). En esencia, esto conlleva un declinar en los retos legales en favor de las acciones de otros niveles de gobierno y una potenciación de los procesos de negociación y acuerdos polítcos. Las reuniones de los primeros ministros nacionales sobre los más variados aspectos son muy frecuentes. Los ministros federales y provinciales responsables de sanidad, finanzas o agricultura, por ejemplo, se encuentran regularmente y han creado una gran burocracia intergubernamental para apoyar estos procesos.

\section{Conflictos de sistemas y leyes básicas}

Durante este período, los temas se centraron en cuestiones de sistemas y politicas públicas. Hasta cierto punto, el conflicto de sistemas sobre las reglas del juego constitucionales en Canadá fueron fruto de las preocupaciones planteadas por varios gobiernos de Quebec. Esta provincia tradicionalmente ha tenido un gobierno separatista, que se opuso a aprobar las enmiendas a la Constitucion de 1982.

Sus exigencias para reformar la Constitución continúan insatisfechas a pesar del intento Meech lake de finales de los ochenta, y del más reciente Charlottetown Accord. En 1994, Quebec ha vuelto a votar a un partido separatista, el Parti Quebecois (P.Q.).

Aunque Quebec históricamente se ha preocupado de prevenir las intrusiones federales en los poderes atribuidos a la provincia por la Constitucion, las discusiones de Meech Lake y el acuerdo de Charlottetown han provisto a Quebec de elementos adicionales para sus intereses específicos. En las discusiones de Meech Lake, Quebec quería ser reconocida como una sociedad distinta, asumir poderes crecientes sobre inmigración, tener derecho de veto sobre las enmiendas constitucionales y compensaciones financieras cuando Quebec opte por no integrarse en los programas nacionales (federales-provinciales) que afecten a competencias provinciales. En las negociaciones de Charlottetown, surgieron demandas adicionales.

Estas incluían, si una provincia lo solicitaba, la retirada del Gobierno Federal de competencias en materias de bosques, minas, turismo, recreo, vivienda, y asuntos municipales y urbanos, en su jurisdicción.

Además, las competencias en asuntos de cultura y formación profesional quedarían dentro de la exclusiva jurisdicción provincial.

El fracaso de estas dos iniciativas se percibe por los "Quebecois" como muestra del rechazo por parte del resto de los canadienses a lo que eran legítimas demandas para reformar la Constitución.

En este contexto, no sorprende que el nuevo partido político, el "Bloque Quebecois" (B.Q.) se constituyera para luchar por la soberanía de Quebec, a un nivel federal. Lo que quizás puede ser sorprendente es que el apoyo que se dio dentro de Quebec al B.Q. fue tan fuerte que hizo surgir la "Oposición Oficial" en el Parlamento federal tras las elecciones de 1993.

Las provincias orientales de Canada han expresado también su preocupación en lo referente a las normas del juego constitucionales. Su punto de vista es que, como consecuencia del principio de representación de la poblacion, la representación de las perpectivas del Canadá occidental en las políticas nacionales ha sido débil en los pasillos de poder de la capital de la Nación. Han sugerido que la Constitución se enmiende de tal modo que el 
Senado de Canadá, como Cámara alta, se someta a los criterios de nombramiento electoral con equidad y efectividad. En otras palabras, han presionado por la igualdad de representación regional en el Senado y una representación regional en el proceso legislativo a nivel federal mayor que la que ha existido en el pasado.

Los años ochenta fueron una década de reforma constitucional, descontento constitucional y el fracaso de acomodar los intereses de las distintas provincias cambiando las "reglas del juego".

No hay duda de que el futuro de la federación desde los ochenta es discutido con mayor seriedad y agilidad que en cualquier momento del pasado.

\section{Conflictos de políticas públicas: del federalismo expansionista a la restricción fiscal}

La segunda área de conflictos gira en torno a las políticas públicas que a menudo reflejan que la división de poderes en la Constiución canadiense no se correspnde con un sistema de comportamientos estancos.

En muchos aspectos el eje de esta área conflictiva es el denominado "federalismo fiscal".

Aunque las primeras subvenciones condicionadas del Gobierno Federal a las provincias se pueden encontrar en 1927, caso de las pensiones a la tercera edad, se conviertieron en un factor relevante del federalismo canadiense a partir de la segunda guerra mundial.

En los años 50 y 60 surgieron varias iniciativas federales de políticas nacionales que conllevaban la intromisión en ámbitos de competencia provincial.

Ejemplos de estas iniciativas fueron el desarrollo del Sistema Sanitario, salud y la educación post-secundaria. Poco tiempo después estas tres políticas fueron definidas como "Fondos para Programas Establecidos" (E.P.F.).

Otro ejemplo que podemos señalar es la asistencia social para los pobres "El Plan Canadiense de Asistencia" (C.A.P.) que se puso en marcha en 1966. Además, se implementaron gran variedad de iniciativas de desarrollo regional, cuyo exponente más reciente es el "Programa Económico de Desarrollo Regional" (E.R.D.P.).

El Gobierno Federal se ampara en que le asiste el derecho constitucional a gastar el dinero en aquello que desee. El atractivo del dinero federal y la popularidad política de estas iniciativas incentiva a los gobiernos provinciales a aceptarlas, compartiendo el coste de los programas (subvenciones condicionadas).

Originalmente el Gobierno Federal aceptó sufragar el 50 por 100 de los costes de este tipo de programas, aunque fueran llevados a la práctica directamente por las provincias, siempre que éstas cumplieran con los estándares nacionales.

Se resistieron pocas provincias, a pesar de sus quejas referentes a que estas iniciativas del Gobierno Federal distorsionaban las prioridades provinciales y hacian transferir fondos provinciales, para ejecutar aquello que Otawa había determinado como prioridades nacionales.

Obviamente fue la Provincia de Quebec la única que optó por no participar en este tipo de programas, al menos, en las primeras etapas. Quebec sostenía que el criterio nacional no sería apropiado para su peculiar población, cultural y lingüísticamente. Estaría, sin embargo, de acuerdo en llevar a cabo programas similares, si el Gobierno Federal proporcionara al Gobierno de Quebec un mayor acceso a los tributos establecidos por el Gobierno Federal en el área de Quebec.

El Gobierno Federal aceptó esta condición y Quebec, aun manteniendo las "apariencias" constiucionales, se encontró con mayor autonomía y libertad respecto a los controles federales, que el resto de las provincias de Canadá.

El informe Dyck (1993) hace constar que ya en 1990 estaban en marcha 206 programas multilaterales y 193 bilaterales de este tipo.

Las transferencias fiscales del Gobiemo Federal a las provincias en 1991-92 alcanzaron la cifra de unos 34.4 billones de dólares, sólo en lo referente a asistencia para el desarrollo regional.

Para 1993-94, este tipo de transferencias se habian incrementado hasta 39.2 billones de dólares, lo que representa aproximadamente el 24 por 100 del total de los gastos del Gobierno Federal (Policy Options 1993).

En muchos aspectos de la realidad canadiense actual, las transferencias fiscales federales son el pilar de las discusiones en torno a la relación federación-provincias.

Ya en 1970 el Gobierno Federal reconocía que los programas de subvenciones condicionadas habían supuesto incrementos masivos en los gastos, sobre los cuales, por otro lado, el Gobierno Federal tenía poco control.

Como resultado del crecimiento del déficit de la Federación, el gobierno tomó medidas para limitar y controlar la tasa de crecimiento de sus transferencias fiscales a las provincias.

La recesión a comienzos de los años 90 elevó todavía más las preocupaciones de las provincias. La recesión redujo los ingresos provenientes de las tasas provinciales y, por el contrario, la demanda de cierto tipo de servicios como los de salud, asistencia médica y social continuaban incrementándose.

Los intentos del Gobierno Federal por controlar su creciente déficit a través del recorte de transferencias fiscales puso a los gobiernos provinciales en una situación muy díficil (De KOven, 1993). 
Al mismo tiempo, se puede argumentar que estas decisiones federales están reduciendo las posibilidades de que el Gobierno Federal pueda controlar los gastos de los gobiernos provinciales en áreas como la de salud, educación post-secundaria y asistencia social. Cuando se iniciaron estos programas en los años $60 \mathrm{el}$ Gobierno Federal podia imponer condiciones estrictas a las provincias y asegurarse que esas condiciones se cumplieran.

Si las provincias no cumplian estas condiciones se recortaban las transferencias fiscales hasta que adaptaran sus programas a los estándares y requisitos nacionales.

Una de las respuestas federales a la crisis fiscal ha sido transferir el acceso a ingresos fiscales adicionales, más que incrementar la transferencia de fondos de los ingresos por tributos federales.

En 1994, por ejemplo, la transferencia de fondos a las provincias en concepto de "Fondos para Programas Establecidos" (E.P.F.) ascendió a la cantidad de 9.05 billones de dólares, mientras que el valor de lo recaudado en concepto de impuestos transferidos fue de 11.5 billones (Canada tax Foundation, 1994).

Si esta dirección de la políica fiscal continúa, la posibilidad de control federal a través del recorte de las transferencias de fondos se irá reduciendo progresivamente, por lo que se dificultará todavía más la homogeneidad nacional en la prestación de servicios en áreas como la de la salud.

El bloque de transferencias anunciado por el Ministro de Finanzas, Sr. Martin, en su reciente discurso presupuestario, ha llevado a alguna autoridad provincial a expresar en privado su creencia de que este cambio va a provocar, a su vez, cambios radicales en las condiciones y requisitos de información impuestos a las provincias por el Gobierno Federal.

En contrapartida a la menor transferencia de fondos, las provincias tendrán mucha más libertad para decidir en qué quieren gastar esos fondos.

\section{¿Existe un papel para la evaluación?}

El federalismo canadiense es complejo, confuso y tiene serios problemas. Canadá se enfrenta a la separación de unas de sus mayores provincias. Financieramente, todos los niveles de gobierno están preocupados en reducir déficits corrientes y niveles de deuda.

Los intereses son cada vez mayores que antes en las negociaciones sobre programas y políticas en las que participan más de un nivel de gobierno.

En este contexto, se puede asumir que la evaluación tendrá un papel útil a desempeñar. Por ejemplo, puede identificar programas de alto coste que deban ser eliminados o sugerir otros que sean más eficientes.
Dados los enormes gastos que llevan aparejados los programas intergubernamentales, la evaluación puede ayudar a generar reducciones de gastos y/o mejoras en estos programas.

Existen otras razones que hacen esperar que los programas intergubernamentales de Canadá sean evaluados.

Originalmente, la política federal de evaluación que empezó a ser operativa a comienzos de la década de los ochenta, requería una evaluación de los programas por ciclos de cinco años (SEGSWORTH, 1990). Aunque los gobiernos provinciales tienen diferentes políticas en lo que a la evaluación se refiere, en el caso de Ontario, se exige que preceptivamente sean evaluados aquellos programas cuyo impacto sea considerablemente incierto (SECSWORTH, 1990). En Ontario, también, los programas sometidos a importantes presiones políticas, con pocas posibilidades de ser modificados, no serían evaluados. En algunos casos, como en los "Acuerdos Económicos de Desarrollo Regional", es obligatoria la evaluación de cada proyecto, evaluación cuyos resultados alimentarán el proceso de decisión para dar por finalizados y/o renovar los acuerdos federales provinciales.

Sin embargo, en la política federal de evaluación de programas existe una gran resistencia a que los programas intergubernamentales sean frecuente y rigurosamente evaluados. La política federal establece que quien encargará la evaluación será el Subsecretario del Departamento y los Subsecretarios se orientan en mayor medida a las cuestiones operacionales.

HaRTLE (1990), por ejemplo, ponía de manifiesto que los aspectos fundamentales de políticas y programas no eran seriamente evaluados, siguiendo los cánones de la política federal de evaluación.

El Grupo de Trabajo sobre Revisión de Programas (1985) y la Oficina del Controlador General (1986) señalaron que el Access to information Act podría haber provocado una tendencia de los departamentos a ocultar o ignorar temas sensibles en la investigación evaluativa.

Los testimonios dados al Comitê Senatorial de Finanzas Nacionales (1990) por los oficiales del Departamento de Pesca, indicaban que los criterios utilizados para seleccionar los programas a evaluar, eran sopesados de manera que las necesidades de los directivos influían del mismo modo que los otros siete criterios juntos.

Como señala SEGSWORTH (1993) uno de los problemas de la política federal de evaluación es que no responde ni a las necesidades del Parlamento ni del público.

Un informe reciente del Auditor General (1993) aporta la evidencia desconcertante de la falta de interés e implicación federal en la evaluación de los programas intergubernamentales. Asimismo, destaca que la evaluación de los programas tiene el potencial de responder a tres acuciantes necesidades de información:

Primero, ayuda a la toma de decisiones para la asignación de recursos que contribuya a controlar el déficit. 
Segundo, ayuda a los canadienses para la determinación del valor obtenido con sus impuestos.

Tercero, capacitar a los servidores públicos, para que asuman responsabilidades por los resultados más que por el proceso. (Pág. 219).

El Auditor General destaca particularmente que los programas más caros son menos susceptibles a la evaluación. Considera que en "programas tales como los de Educación Post-secundaria, Igualación y Estabilización fiscal y Contribuciones al Seguro de la salud, se deben establecer nuevos mecanismos en orden a fomentar la consulta y cooperación con los gobiernos provinciales de cara a asegurar que las brechas existentes en el proceso evaluador queden cubiertas".

De hecho, el interés del Auditor General sobre la evaluación de programas, llega al punto de señalar: "Si el actual sistema no resultase operativo, el Parlamento puede tomar en consideración soluciones más radicales en aras a obtener más información relevante, fiable y a tiempo".

Es importante tener en cuenta que la crítica del Auditor General sobre la evaluación de programas se hace desde el punto de vista del Gobierno Federal. Esto se subraya claramente en la discusión del informe que define a los interesados en esta materia: Gobierno Federal, Parlamento, Agencias Centrales y Departamentos Federales.

La utilización de la evaluación está igualmente centrada en los "actores" federales: Parlamento, Ministros, Subsecretarios y gerentes de los programas a nivel federal. Se hace muy poco énfasis en la colaboración intergubernamental de la evaluación de los programas cuando ambos niveles tienen claros intereses en la misma.

Aunque es cierto que extensas áreas de los programas federales como las transferencias a las provincias de los seguros sanitarios y educación post-secundaria, no han sido evaluadas, en los pequeños sub-programas, bajo acuerdos de costes compartidos, sí se incluyen requisitos de evaluación.

Para algunas provincias, el cumplimiento de estos requisitos de evaluación fue la primera experiencia a nivel provincial de lo que es la evaluación de programas.

La ampliación de Los fondos federales para el Programa de Asistencia civil (C.A.P.), por ejemplo, se ofreció a las provincias a comienzos de los años 80 , incluyendo la evaluación como una de las condiciones para acceder a los fondos federales.

La exigencia de la evaluación de programas vinculados a proyectos experimentales y proyectos basados en la comunidad en campos como la prevención del crimen, programas para mayores, servicios a las víctimas y salud comunitaria, fue probablemente una línea importante de trasmisión para la difusión de la práctica de la evaluación en los gobiernos provinciales y municipales de Canadá.
A.W. Johnson (1992) concluye que se han implementado pocos sistemas de evaluación para grandes programas, debido a las consecuencias políticas que se derivarían de la propia evaluación tanto para el federalismo canadiense como para el sistema parlamentario.

Canadá tiene una antigua institución, la "Royal Commision" que ha sido utilizada para la revisión de grandes programas nacionales y temas de una importancia política crítica. JOHNSON escribe:

"... el problema fundamental de la efectividad del régimen de evaluación tal como se ha desarrollado... es su falta de compatibilidad con el sistema parlamentario en el que se ha de implantar. La esencia de este sistema es que es político y configurado entre adversarios. (P. 26)".

JOHNSON argumenta que el encontrar estrategias alternativas para evaluar las politicas y programas de gran alcance mantendria en el régimen eficaz de evaluación sólo aquellos programas de incidencia politica minima, dado su gran componente administrativo.

Esta línea de pensamiento hace surgir cuestiones que van más allá del propósito de este artículo. Es útil, sin embargo, contrastar los "grandes y pequeños" programas intergubernamentales y reconocer que la experiencia canadiense más común, en lo que se refiere a la evaluación intergubernamental, ha sido la evaluación de los "pequeños" sub-programas resultantes de los acuerdos federal-provinciales. Nuestros dos casos de evaluación corresponden a programas de tamaño medio, aunque la ayuda legal es, en cierto modo, mayor y tiene un status de programa diferente que los proyectos experimentales insertos en el Programa de Oportunidades de Empleo. Estos dos casos muestran alguno de los caracteres distintivos de la evaluación intergubernamental en Canadá. Reflejan la realidad canadiense, las políticas públicas altamente politizadas no están normalmente sujetas a evaluación según las actuales políticas evaluadoras tanto federales como provinciales.

\section{Juego paralelo: la evaluación del programa de oportunidades de empleo (E.O.P.)}

El Programa de Oportunidades de Empleo era, en realidad, una serie de programas piloto desarrollados por el Ministerio de la Comunidad y Asuntos Sociales de Ontario a comienzos de la década de los 80 .

La meta básica de cada uno de estos programas era dotar de una serie de servicios de distinto tipo a los destinatarios de la 
asistencia social, a fin de capacitar a estas personas para encontrar empleo y dejar de depender de la asistencia del Estado. Cada programa fue diseñado para un grupo destinatario específico. En el cuadro 1 se presenta el perfil de cada una de las iniciativas:

El E.O.P. era en muchos aspectos el candidato ideal para la evaluación intergubernamental. Era razonablemente caro, coste total de 42.7 millones de dólares entre 1987-88. Los fondos del E.O.P. provienen de dos fuentes principales: los Gobiernos de Canadá y Ontario. Los fondos federales del Plan de Asistencia de Canadá (C.A.P.) que es un programa de costes compartidos establecidos en 1966. "Bajo la cobertura del Plan de Asistencia de Canadá, Otawa sufragaba la mitad de los fondos requeridos para casi todos los programas provinciales y municipales encaminados a la asistencia social y al bienestar de los necesitados" (DYck, 363-364).

La financiación federal-provincial y la relación entre los programas se pueden describir como una interacción multilateral que se unifica en el marco creado federalmente y denominado C.A.P., a pesar de que la decisión de evaluar se toma unilateralmente.

El E.O.P. implicaba algunas actividades que se solapaban potencialmente con programas operados por el Departamento Federal de Empleo e Inmigaración. Además, los mecanismos de implementación de los programas requerian la participación de los gobiernos municipales y del Ministerio provincial de la Comunidad y Asuntos Sociales, así como de multitud de agencias de servicios sociales.

La evaluación del E.O.P. fue una evaluación cara que se llevó a cabo en el período 1984-86.

Se completaron trece estudios diferentes que intentaron examinar tanto la eficacia como aspectos de implementación de los programas. Uno de los estudios implicó un mayor seguimiento de las Iniciativas de Apoyo al Empleo y la evaluación del mismo. Otro de los estudios implicaba la revisión de los distintos informes relativos a los programas de empleo destinados a receptores de asistencia social. El coste total de la evaluación fue de más de un millón de dólares.

La decisión de evaluar el E.O.P. surgió inicialmente de una reunión de pasillo entre el gerente de un programa y un miembro del staff de evaluación del Ministerio.

El evaluador sugirió que los proyectos pilotos como el E.O.P. deberían ser evaluados y el gerente aceptó. Esta recomendación fue asimismo aceptada por ejecutivos de más nivel dentro del Ministerio.

El Ministerio provincial también decidió que no hubiera implicación ni del Gobierno federal ni del municipal ni de otras

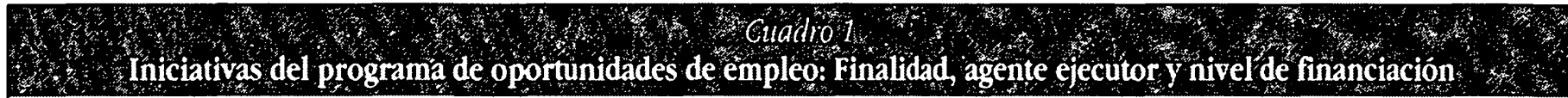

Programa de iniciativas de apoyo al empleo

Aportar asesoría pre-empleo a padres solteros. A ejecutar en la provincia por los gobiemos municipales (9.9 millones de dólares en 1987-88)

\section{Programa de empleo juvenil}

Proporcionar asesoría pre-empleo por un mínimo de tres meses a personas entre 16 y 24 años que hayan estado en la asistencia social al menos tres meses: A ejecutar por gohiernos municipales (1.1 millones de dólares en 1987-88)

\section{Programa Municipal de trabajo}

Aportar experiencia laboral y ayudar a encontrar trabajo a tiempo completo en los receptores de la asistencia social. A ejecutar por gobiernos municipales (1.3 millones de dólares anualmente).

\section{Programa de Empleo de Servicios Sociales}

Para facilitar empleo a tiempo completo por 12 meses al menos a padres solteros y a individuos incapacitados, en agencias del C.A.P. con costes compartidos. Puesto en práctica por el Ministerio provincial de la Comunidad y Servicios Sociales (16.3 millones de dólares).

\section{Experiencia de trabajo en verano a tiempo parcial}

Aportar experiencia de trabajo a personas de entre 16 y 20 años destinatarias de la asistencia social o a personas protegidas por las sociedades de ayuda a la infancia. Durante el verano y durante el año escolar en Agencias Comunitarias sin ánimo de lucro. A ejecutar por el Ministerio provincial de la Comunidad y Servicios Sociales (4.5 millones de dólares en 1987-88).

\section{Programa de Apoyo Comunitario a la juventud}

Aportar asesoría gerencial práctica y servicios relacionales a personas de entre 15 y 20 años que vivan independientes y tengan dificultades. A ejecutar por las Agencias de transferencias ministeriales (4.1. millones de dólares en 1987-88).

\section{Programa de preparación para la independencia}

Proporcionar "técnicas" para desenvolverse en la vida y formación pre-empleo a personas entre 15 y 20 años. A ejecutar por las Agencias de transferencias del Ministerio (2.4 millones de dólares).

\section{Componente Residencial de Futuros}

Aportar asistencia en la vivienda y apoyo a personas de entre 16 y 24 años incluidas en el Programa de Fururos. A ejecutar por las Agencias de transferencias del Ministerio ( 3.1 millones de dólares anualmente). 
agencias de implementación, en el diseño y gestión de los esrudios de evaluación.

Otros interesados en el asunto, diferentes del Ministerio de la Comunidad y de Asuntos Sociales fueron expresamente excluidos, a pesar de la recomendación en contra del staff de evaluación del Ministerio.

La decisión de la alta direccción reflejaba un interés políticoburocrático y también es significativo el hecho de que los fondos para los estudios de evaluación provinieran exclusivamente del presupuesto del Ministerio.

Se formaron dos Comités para el diseño del desarrollo de la evaluación para supervisar el proceso evaluador.

El Comité Asesor lo componían el Subsecretario Adjunto, altos oficiales del Ministerio y miembros del staff del área. El Comité de Trabajo lo componían gerentes del programa de la oficina central y miembros del staff de evaluación del Ministerio. Este grupo se reunió con frecuencia para desarrollar los términos de referencia para los estudios de evaluación. Sus recomendaciones se aprobaban seguidamente por el Comité Asesor.

El Comité Asesor se reunió en raras ocasiones a efectos de dirimir sobre preocupaciones específicas expresadas por el Comité de Trabajo y para aprobar solicitudes adicionales de fondos cuando era necesario.

Tomando en consideración que todas las personas implicadas en el diseño de los estudios de evaluación provenían del Ministerio, no nos debe sorprender que hubiera muy poca controversia en lo referente a cuestiones fundamentales de evaluación o al ámbito de la misma.

En otras palabras, los evaluadores se limitaban al campo de interés del Ministerio de la Comunidad y Asuntos Sociales. Temas como el posible solapamiento o complementariedad con los programas ofrecidos por el Departamento de Empleo e Inmigración de Canadá fueron ignorados.

Una vez que el Comité de Trabajo había desarrollado los términos de referencia para las distintas evaluaciones, se solicitaron sugerencias externas y se invitó a consultores externos a hacer propuestas. Los consultores se unieron al Comité para trabajar conjuntamente. Las reuniones fueron comunes. Hay que subrayar que los consultores no tenían acceso a las bases de datos federales ni a la mayoría de las provinciales por razones de confidencialidad protegida, tanto por la legislación federal como por la provincial.

Los participantes de los programas a evaluar firmaron los informes de utilización de datos y junto con el staff del programa aportaron la mayor parte de la información necesaria para completar los estudios. Una vez finalizados éstos se enviaron a los oficiales del Ministerio, a todas las agencias de implementación, a los gobiernos municipales y a los departamentos federa- les de Empleo e Inmigración y de Salud y Bienestar. Los informes generaron un interés considerable en algunas jurisdiccio nes, particularmente en las provincias occidentales de Canadá.

A pesar del carácter intergubernamental del programa, la variedad de fondos y el sustancial coste del mismo, se instrumentaron pocas posibilidades de utilizar los resultados de la evaluación.

Muchos de los estudios concluyeron que los programas eran bastante ineficaces, especialmente los destinados a la juventud.

Naturalmente se despertó alguna reacción contra estas conclusiones por parte de individuos que tenían intereses creados en la continuidad de los programas. Al final éstos triunfaron en la mayoría de los casos y los programas ineficaces continuaron operando.

Una de las explicaciones de este fenómeno la podemos encontrar en la gran rotación de ejecutivos dentro del Ministerio. Tan pronto como demostraron su interés en la evaluación de los programas y sus resultados, y determinado que continuarían, los ejecutivos eran promovidos o trasladados a otras responsabilidades. El resultado es que no se tomaban las decisiones claves para terminar o modificar las iniciativas.

Además, la evaluación tampoco fue utilizada de un modo más indirecto. El Gobierno Federal, por ejemplo, no se valió de los informes para defender ante las provincias el que éstas no estuvieran utilizando las transferencias federales eficientemente. Las provincias tampoco utilizaron sus informes en su debate con el Gobierno Federal acerca de los recortes en las transferencias federales a Ontario amparadas por el C.A.P.

El Gobierno Federal tampoco exploraba su propia evaluación y tampoco participaba directamente en el control de estos programas. El contexto evaluador no se podía describir como algo competitivo entre los niveles de gobierno.

\section{Cooperación sin consecuencias en las políticas públicas: la evaluación de la asistencia jurídica en Ontario}

A diferencia del caso previo, la decisión de poner en práctica la evaluación del Plan de Asistencia Jurídica de Ontario (O.L.A.P.) fue tomada en el contexto de un marco evaluador nacional (Poet, 1993). A principio de los 80 el Departamento Federal de Justicia recibió la aprobación, a nivel de Gobierno, para la evaluación de los servicios de asistencia jurídica financiados a nivel federal y administrados por las diez provincias canadienses. 
La implementación de este mandato requería la cooperación del nivel provincial y Nova Scotia fue la primera provincia en participar (Poel, AuCoIn y CONWWy, 1980; POEL, 1983).

Esta iniciativa fue seguida con colaboración federal-provincial en New Brunswick, British Columbia, Manitoba, Saskatchewan y Alberta. El O.L.A.P. y el Ministerio de Justicia de Ontario se sumaron a esta serie de evaluaciones a raíz de un acuerdo al que se llegó en 1988 (ver ABT1991).

Para 1988, el Justice Bureau of Review había ya adquirido una experiencia considerable en la evaluación de los servicios de asistencia jurídica y tenía el compromiso de realizar la evaluación en el marco que había sido ya implementado en todas las provincias de Canadá, excepto en las dos más pequeñas (Prince Edward Island y Newfoundland) y en la mayor (Ontario). Este marco se había polarizado en torno a las cuestiones de evaluación del acceso, la calidad y los costes de los servicios de asistencia jurídica.

Las relaciones federaciones-provincias son más complejas en las políticas del área de justicia y en la ejecución de los servicios de asistencia jurídica que en el campo de la formación profesional y de apoyo al empleo.

Los programas E.O.P. de Ontario podían ser implementados (y evaluados) sin significativas referencias a sus programas homólogos desarrollados también en Ontario pero con fondos federales. Mientras las provincias tienen la responsabilidad constitucional de la Administración de Justicia (incluidos los servicios de asistencia jurídica), la legislación penal y otros estatutos legales (ej., food and drugs, divorcio) son competencia federal. Además, los jueces de los Tribunales Superiores Provinciales son designados por las autoridades federales, mientras que los jueces de familia e instancias penales inferiores son designados por las autoridades provinciales. El entrecruzamiento de las responsabilidades constitucionales en los asuntos de justicia es una presión obvia a la cooperación en las políticas, programas y evaluación entre niveles de gobierno.

Los primeros acuerdos federación-provincias para compartir costes en la ejecución de asistencia jurídica penal fueron más generosos que los programas sociales diseñados bajo la cobertura del C.A.P., pagando el Gobierno Federal el 90 por 100 de los servicios de asistencia jurídica penal.

Los primeros estándares nacionales requeridos por el Gobierno Federal a los planes provinciales se centraban en áreas como la cobertura y la elegibilidad por parte del cliente. La elección de los mecanismos de implementación quedaba al arbitrio de las provincias, y éstas podían requerir al destinatario del servicio legal para que contribuyera en los costes del servicio.

Dentro de la interacción federación-provincias, la política federal referente al acceso y servicios es una constante a lo largo de las 10 provincias, con las variaciones resultantes de las áreas en las que las provincias tienen mayor discrecionalidad, bien en términos de implementación de los servicios o, en las provincias más ricas, en la prestación de servicios adicionales más allá de las exigencias federales.

Por diversas razones, a los oficiales del Ministerio de Ontario y a los gerentes de más nivel del programa L.A.P. les preocupaba que el Departamento Federal de Justicia pudiera utilizar los resultados de la evaluación para argumentar y defender un cambio en cómo se deberían de prestar los servicios de asistencia jurídica. Un cambio del sistema predominante de Ontario, del Judicare system, en el que los servicios de asistencia jurídica son prestados por abogados particulares a los clientes que acreditan un certificado hacia otro sistema, el staff lauyer system, en el que los servicios son prestados por abogados empleados por el Plan de Asistencia Jurídica. Las cuestiones claves en el debate entre estos dos tipos de sistemas son el coste relativo de los mismos, el principio de libre elección de abogado y la calidad de los servicios.

El diseño de la evaluación, según fue negociado entre colaboradores federales y provinciales quedó limitado al judicare 0 certificado del O.L.A.P., quedando excluidos así los componentes del staff lawyer system que jugaban un papel importante en las clínicas especializadas de la Comunidad y en la división de investigación. También las cuestiones de costes fueron limitadas al modelo de predicción de costes que operaba dentro de los parámetros existentes ligados a servicios basados en certificados. De esta manera, el nivel inferior del gobierno estaba capacitado para negociar algunos aspectos de la evaluación no incluidos en el diseño general de evaluación. El informe final se organiza primordialmente en torno a las prioridades federales de evaluación, a saber, accesibilidad, calidad de servicio, análisis financiero y de costes, y análisis de gestión.

El coste total de la evaluación de Ontario estuvo por debajo de 500.000 dólares. En la mayoría de las otras provincias el Departamento federal a través del Bureau of Review, pagaba la mayor parte de los costes de los contratos de los consultores para las evaluaciones de campo. Las provincias contribuian básicamente a través de contribuciones en especie (tiempo del staff, instalaciones) 0 absorbiendo gastos reales en sus existentes presupuestos operativos. En comparación con otras provincias, Ontario probablemente, contribuyó en una mayor proporción al presupuesto de evaluación (salvo Quebec que lo hizo por sí misma sin cooperación federal).

Los costes de evaluación fueron relativamente bajos en relación con el presupuesto de la O.L.A.P. que fue de unos 114 millones de dólares en el ejercicio 1987-88 y que se incrementó drásticamente en 1991-92, llegando a los 271,5 millones de dólares, por el impacto que tuvieron una decisión de los tribunales (R.V. Askor), nuevos procedimientos de determinación de refugiados, incremento en el número de agresiones a las mujeres, y persecución de la droga y perjuicios derivados de la conducción. 
Las negociaciones que se llevaron a cabo en aras a la consecución del acuerdo de evaluación y el plan final de la evaluación estuvieron dominados principalmente por intereses profesionales. Los más importantes evaluadores fueron los profesionales del Departamento Federal de Justicia, los del Ministerio de Justicia de Ontario, los del O.L.A.P. y los representantes de la Sociedad legal del Alto Canadá (asociación profesional) que administra el Plan de la Provincia y contribuye financieramente a la operación. Estos profesionales, en representación de las organizaciones más influyentes, actuaron con considerable continuidad y autonomía.

En la mayoría del resto de las provincias el nivel federal podía dominar el proceso evaluador por su importante contribución financiera y por ser la primera fuente de expertos evaluadores. Esto no fue tanto en Ontario, pues los recursos, tanto financieros como humanos, del gobierno de la provincia y sus agencias fueron muy cuantiosos y significativos.

Los políticos como tales, sin embargo, no tuvieron un peso destacable en el proceso evaluador. Es interesante saber que, durante el transcurso temporal de esta evaluación, tres gobiernos provinciales diferentes ostentaron el poder, primero los conservadores progresistas, seguidos por un gobierno liberal y, finalmente, un partido Social Democrático, "The New Democratic Party" (N.D.P.).

El gobierno del N.D.P. estaba en el poder cuando el informe final de la evaluación salió a la luz. Por un tiempo, el nuevo gobierno no se hizo eco del informe y, en las mismas fechas, por razones de restricciones financieras, empezó a haber presiones hacia un cambio de sistema al modelo Staff Lawyer (ONYSHKO, 1991). Un cambio en el entorno político puede ser un gran problema en las evaluaciones intergubernamentales. Un cambio de gobierno puede suponer un "nuevo socio" para el ejercicio evaluador, un socio que puede continuar o no con los compromisos previos de una evaluación en marcha.

La medida en que un cambio gubernamental influye en el proceso evaluador o el uso que se haga de sus resultados dependerá, en gran parte, de que el proceso evaluador o sus resultados sean utilizables políticamente 0 , por el contrario, queden en la esfera de dominio de los gerentes. En este caso particular, la evaluación de la O.L.A.P. parece menos politizada a nivel federal y, en cambio, más utilizable políticamente en el escalón provincial.

En contraste con los resultados de la evaluación del E.O.P., el tono general del informe del A.B.T., Associates of Canada, fue favorable como se refleja en la siguiente conclusión:

Tomados en su conjunto, los datos recogidos para esta revisión presentan un aspecto general positivo del rendimiento del Plan. El Plan está suponiendo un servicio generalmente accesible. Tanto la administración del mismo como los criterios empleados para evaluar a los posibles usuarios del servicio están bajo revisión. El Plan toma conciencia y es responsable de un número de segmentos de la población que requiere atención especializada.

El Plan debería ser elogiado por sus logros hasta la fecha e impulsado para construir sobre la base de estos éxitos en el futuro.

(A.B.T., 283)

Las recomendaciones del informe sugerían caminos para mejorar la eficiencia administrativa del Plan y la calidad de los servicios. También se recomendaba una reestructuración de su organización hacia un diseño regional, se sugerían campañas de información pública para algunos clientes y grupos de referencia. La mayoría de las recomendaciones fueron centradas en la gestión del programa de asistencia jurídica y no en temas políticos.

El informe eludió pronunciarse directamente sobre la expansión o disminución de los servicios del staff lauyer del plan, y virtualmente no dice nada sobre la previsión de menos servicios. Como resultado de lo antedicho, el informe fue bastante irrelevante en la agenda del nuevo gobierno N.D.P., el cual rápidamente adquirió un enfoque miope en los recortes del presupuesto.

Las cuestiones del programa contampladas en la evaluación fueron importantes para afinar el diseño de una gran organización cuya capacidad de gestión había quedado rezagada con el crecimiento del programa. Estos asuntos no fueron de inmediato puestos en la agenda ni de los políticos federales ni provinciales. Después de tres años de esfuerzo investigador las interrogantes de la evaluación fueron respondidas, pero no hubo un feed-back rápido del ejercicio evaluador.

Durante y después del período evaluador ambos niveles de gobierno tomaron decisiones significativas sobre financiación e implementación de la asistencia jurídica, con pocas alusiones o referencias al proceso de evaluación que se estaba siguiendo 0 a sus conclusiones, una vez finalizado.

Los gastos del Gobierno Federal en aistencia jurídica se incrementaron en cifras poncentuales de dos dígitos durante este período (una media del 19 por 100 anual en Ontario), "taponando" su contribución a los programas en Ontario, Alberta y British Columbia las tres provincias más ricas de Canadá. El gobierno provincial del N.D.P. presionó según su interés en el sistema alternativo de ejecución, staff lawyer clinics y retrasó los incrementos programados en el cuadro de tarifas determinante de los honorarios de los abogados particulares que actuaban previa presentación por parte del cliente del certificado acreditativo del derecho a la prestación del servicio. El informe del A.B.T. Associates no trataba de las cuestiones del staff lawyer y recomendaba mejoras en los honorarios de los abogados particulares. 


\section{Aplicaciones de la evaluación intergubernamental}

El I.I.A.S., grupo de trabajo sobre evaluación de políticas y programas ha concluido en sus decisiones que la característica más típica de la evaluación intergubernamental es, probablemente, el hecho de que contiene una gran parte de ingredientes políticos (TOULEMONDE y REIPER, en imprenta). Esto no parece ser el caso de Canadá ni generalmente ni especialmente en los dos casos estudiados.

Estos casos reflejan que en la realidad canadiense las políticas y programas "altamente politizados" no se sometan normalmente a una evaluación formal bajo una política federal o provincial de evaluación.

Existen tres posibles explicaciones de este fenómeno:

1) El enfocar el uso de la evaluación para la gestión de programas, uso instrumental común de la evaluación en gobiernos de un solo nivel.

2) La fijación de la agenda de la evaluación intergubernamental por parte del Gobierno Federal donde los programas multilaterales se unifican a nivel federal.

3) El uso de los mecanismos de evaluación alternativos (grupos de trabajo y la Royal Commission) para políticas públicas y programas que tengan un marcado carácter político.

\section{Definiciones de la evaluación intergubernamental}

TOULEMONDE y REIPER definen la evaluación intergubernamental como un proceso en el que dos o más niveles de gobierno están implicados en una o más fases del proceso evaluador, p.e., desde la decisión de evaluar hasta su utilización. Esta definición es problemática porque deja un vacío en la práctica canadiense. En el caso del E.O.P., una parte significativa de los fondos para los programas provienen del Gobierno Federal pero los programas fueron creados y administrados por el gobierno de Ontario. La evalución del E.O.P. fue llevada a cabo unilateralmente por Ontario sin ninguna injerencia federal, aunque, en algún momento, los resultados de la evaluación puedan jugar un papel en las discusiones federación-provincias del Plan de Apoyo de Canadá.

La definición también ignora situaciones competitivas de las políticas públicas entre dos niveles de gobierno. Por ejemplo, en el caso de Northern Ontario, el Ontario Heritage Fund (gobierno provincial) y el F.E.D.N.O.R. (gobierno federal) tienen virtual- mente idénticos mandatos en la misma área geográfica y, por lo tanto, compiten el uno con el otro.

Una evaluación reciente del F.E.D.N.O.R. por parte de sus clientes lo califica como un vehículo más eficiente que el Heritage Fund para apoyar sus iniciativas para la diversificación económica de la región.

Estos ejemplos ilustran una potencial área gris en nuestra comprensión de las políticas intergubernamentales y su evaluación.

En el primer caso, aunque la política es intergubernamental, al menos en parte, la evaluación no lo es. En el ejemplo de F.E.D.N.O.R., por las definiciones de los editores, ni las políticas ni las evaluaciones son intergubernamentales.

Al mismo tiempo, estas políticas reflejan interrelaciones federales, provinciales y regionales desde una perspectiva intergubernamental. Por no contemplar estas evaluaciones desde una perspectiva intergubernamental, ciertos matices se pueden perder como:

* El fracaso de la política de evaluación existente y/o de la exigencia de responsabilidades. En el caso del E.O.P., al Gobierno Federal parece que no le concierne el uso eficaz de su contribución financiera. En el ejemplo de F.E.D.N.O.R., la evaluación ignora asuntos como las duplicidades, la confusión de clientes y ciudadanos y la concurrencia entre programas federales y provinciales.

* El fracaso en asegurar una cobertura adecuada y extensa de la evaluación útil para políticas y programas intergubernamentales. Si aplicamos la definición "estrecha" de evaluación dada previamente sólo uno de los dos casos examinados en este artículo constituiría una evaluación intergubernamental, que sería la evaluación del O.L.A.P. El caso de E.O.P. se consideraría simplemente como la evalución de un solo nivel de gobierno. Una perspectiva así pierde complejidad, sutileza y se aparta de la realidad dinámica de la Política y políticas públicas de Canadá.

En esencia, el tema es si el proceso evaluador y la política a evaluar deben ser intergubernamentales, si el proceso evaluador debe ser "intergubernamental" o si la política o programa a evaluar deben ser "intergubernamentales". Desde nuestro punto de vista, la última alternativa es la más útil. Incluiría tanto la iniciativa de evaluación del O.L.A.P. como la del E.O.P.

\section{El uso gerencial de los resultados de la evaluación}

Los resultados de la evaluación del O.L.A.P. y las iniciativas del gobierno de N.D.P. en Ontario, a pesar del informe del A.B.T. Associates, pueden ajustarse al análisis de A.W. JoHnson, 
p.ej., la evaluación efectiva no es compatible con los ingredientes políticos del sistema parlamentario $y$, por lo tanto, se debe utilizar primordialmente como instrumento de gestión. Dos de las decisiones más políticas que el gobiemo de Ontario podía haber tomado serían:

a) Por un lado la de pagar menos dinero a los abogados particulares cuando prestan servicios de asistencia jurídica, mediante el retraso en los aumentos de tarifas.

b) Por otra parte, seguir presionando a favor de la alternativa del staff lawyer en contra del judicare model, en todo lo relativo a servicios de asistencia jurídica.

De hecho se tomaron estas dos decisiones sin tener en consideración los resultados de la evaluación.

El uso real del informe de evaluación del O.L.A.P. se ha centrado en la gestión del programa sin alterar lo más mínimo la política en sí misma o las decisiones políticas. Ontario recibió varias sugerencias respecto a mejoras administrativas y de reestructuración. Al Gobierno Federal le llegaron recomendaciones en áreas como el acceso al programa y conocimiento del mismo y se le aseguró que el Plan, en conjunto, estaba consiguiendo una buena calidad en la prestación del servicio en relación con la contribución financiera.

El uso de las evaluaciones del E.O.P. era potencialmente muy político, dados los resultados negativos que en general se obtuvieron y que la evaluación puso de manifiesto. En este caso, sin embargo, el férreo control al que fueron sometidos los participantes en el proceso evaluador implicó que cualquier conflicto potencial sería evitado.

El E.O.P. no tuvo que responder a las acusaciones de ineficacia ni a los cargos de duplicidades en programas federales paralelos. El círculo de la evaluación se limitó a un solo nivel y excluyó a los interesados de otros niveles de gobierno que hubieran podido utilizar los resultados de la evaluación para criticar el E.O.P.

El reciente informe del Auditor General de Canadá (1993) muestra que, en primer lugar, las evaluaciones federales se utilizan para la comprensión del programa y para la modificación del mismo en segundo lugar.

En tercer lugar, se utiliza a efectos de la reforma del programa, la evaluación y raramente sirve para acabar con el mismo.

La comprensión y modificación de los programas no son usos triviales de la evaluación y pueden representar no sólo un uso inmediato de la misma sino también una utilización a largo plazo.

La experiencia evaluadora de Ontario y su informe, por ejemplo, muestra como a través del Plan se pueden mitigar los actuales desequilibrios regionales en lo que concierne al acceso a la asistencia jurídica. También contribuye a ensanchar el acervo de conocimiento de los servicios de asistencia jurídica en Canadá, que comenzaron a raíz del informe de la evaluación del programa en Nova Scotia.

Las posibles áreas susceptibles de una reforma profunda en el O.L.A.P. o la virtual terminación de las iniciativas E.O.P. son usos menos probables de la evaluación, dado el contexto político de los dos programas. En el ámbito intergubernamental, se llega a la conclusión de que difícilmente los resultados de la evaluación pueden conducir a decisiones que impliquen reformas significativas de los programas.

Hasta cierto punto, nuestros dos casos de evaluación encajan en un "modelo gerencial" de evaluación intergubernamental como fue desarrollado por TOULEMONDE y REIPER (en imprenta).

La cuestión, sin embargo, es más gerencial que la definida por TOULEMONDE y REIPER. Ellos sugirieron como tema central el siguiente: chasta qué punto la política o programa intergubernamental tiene éxito?, y si matizamos aún más añadiríamos: ¿en qué podemos modificar, para mejorarlo, un programa que básicamente es bueno?

Esta fue la dirección de la evaluación del O.L.A.P. y de las que se implementaron en otras provincias. Hubo diferentes prioridades en las distintas provincias y, asimismo, cambios de énfasis a lo largo de los 10 años que duró la evaluación. La presencia del Gobierno Federal como actor clave en cada evaluación de la asistencia jurídica trajo, sin embargo, una cierta homogeneidad que posibilitó la comparación entre provincias de los resultados básicos de las distintas evaluaciones provinciales, a saber, acceso al servicio, calidad del mismo y eficiencia.

La serie de evaluaciones de la asistencia jurídica también tuvo como consecuencia un cierto aprendizaje sociológico. Aunque existió conflicto en algunos puntos, hubo un consenso general sobre la necesidad de que el sistema de justicia canadiense debe prestar de alguna forma un servicio de asistencia jurídica. En este sentido, los distintos puntos de vista profesionales en este campo no son más que conflictos de poca importancia. Las evaluaciones se enfocaron más lejos de la simple constatación del éxito o fracaso.

El status de los programas de asistencia jurídica, según los "requerimientos" del sistema de justicia canadiense, es significativamente diferente del de los programas E.O.P., que se configuran como proyectos "piloto" provisionales.

El status de los programas se debe considerar en las evaluaciones intergubernamentales. La comparación entre estos dos casos en este sentido puede haber sido menos clara dada la poca implicación intergubernamental en la evaluación del E.O.P. El perfil de su evaluación es bastante diferente del del O.L.A.P. En la evaluación del E.O.P. el nivel provincial controlaba todas las actividades, salvo el uso de los resultados. Este perfil evaluador hizo más fácil el consenso en el diseño de la evaluación y también permitió ignorar algunos asuntos importantes de sola- 
pamiento con programas federales alternativos. En la evaluación del O.L.A.P., la mayoría de las actividades evaluadoras fueron compartidas por ambos niveles, con el solo dominio de la contribución federal en algunos puntos de la agenda y en la financiación económica. El diseño de la evaluación del O.L.A.P. estrechó el ámbito de la investigación y, aun así, hubo duras negociaciones para llegar al diseño definitivo.

La discusión del modelo de "responsabilidad" parece estar equivocadamente dirigido en el sistema parlamentario de Canadá (y Gran Bretaña, etc.), donde el gobierno es responsable ante el Parlamento (o la asamblea legislativa provincial), más que directamente ante la sociedad.

Esta es la distinción básica entre gobierno responsable y representativo.

El hecho de que la evaluación del E.O.P. pudiera encontrar los programas enormemente ineficaces, y así y todo se pudieran mantener los resultados de la evaluación ignorados, sugiere que no había ventaja política para los partidos de la oposición en utilizar estos resultados. Similarmente, los buenos resultados de la evaluación del sistema de asistencia jurídica fueron de interés para los funcionarios y abogados que gestionaban el Plan, pero no para los partidos de la oposición o los ciudadanos, en general.

\section{Política de evaluación en Canadá sin referencia al I.G.E.}

La relación entre la evaluación de programas y responsabilidad, según expuso A.W. JoHnson (1992), subraya los frecuentes desacuerdos en Canadá acerca de la evaluación de programas ante el Parlamento.

Aceptamos su sugerencia, ya discutida anteriormente, de que en el contexto político del Parlamento se hace difícil el uso de cualquier evaluación a fin de una revisión de gran alcance de la política pública en cuestión, intergubernamental o no.

Igualmente, contextos en los que las relaciones intergubernamentales llevan aparejadas cuestiones de políticas públicas o de política son, probablemente, contextos en los que la evaluación de programas, en el mejor de los casos, puede aportar información útil y, en el peor, puede ser poco "apropiada" para la política del momento.

En mayo de 1994, el gobierno de Canadá aprobó una nueva política de revisión de programas, auditorías internas y evaluación, (Treasury Board 1994). En el contexto de la evaluación intergubernamental, esta nueva política es más interesante por lo que no incluye que por lo que contiene. Quizás como respuesta a las preocupaciones expresadas por el Auditor General, la nueva política enfatiza la necesidad y responsabilidad del Treasury Board Secretariat en la coordinación e iniciativa de "revisiones ocasionales sobre asuntos multidepartamentales y de amplitud gubernamental". Sin embargo, la nueva política de evaluación no dice nada respecto a la revisión o evaluación de políticas y programas intergubernamentales. Refleja lo que nuestros casos prácticos han ilustrado:

* Las políticas federales y provinciales de evaluación no incluyen formalmente previsiones para la evaluación de políticas y programas intergubernamentales.

* Como las políticas evaluadoras existentes tienen a un director adunto (a un burócrata senior) como cliente de la inmensa mayoría de las evaluaciones, esto hace que los estudios de evaluación reflejen más problemas de gestión que de la política stricto sensu.

* Las evaluaciones intergubernamentales, de existir, reflejan los acuerdos ad boc de qué se evaluará, qué cuestiones se plantearán, cómo se llevarán a cabo los estudios y quién debe participar en el proceso evaluador.

* Los principales programas y políticas intergubernamentales no están sujetos a evaluación, según las actuales políticas de evaluación, tando federales como provinciales.

Esta política federal revisada es consecuencia de la tremenda sensibilidad políica del gobierno de Canadá hacia la dinámica del federalismo y respecto de las dificultades que entraña el que unilateralmente el gobierno de la nación acometa una evaluación de los grandes programas federal-provinciales.

\section{Notas}

Articulo traducido por M.' Jesús Laguna HuRtado.

* Profesor del Departamento de Ciencias Políticas de la Universidad de Laurentian, Ontario, y Editor de la Revista Canadiense de Evaluación de Programas.
- Profesor de la Escuela de Administración Pública de la Universidad de Dalhousie, Nova Scotia. 


\section{Bibliografia}

Auditor General of Canada (1993). Annual Report. Otawa: supply and Services. Abt Associates of Canada (1991). Comprebensive Review and Evaluation of tbe Centificate Component of the Ontario Legal Aid Plan. Prepared for the Ontario Ministry of the Automey General and the Federal Department of Justice.

Canada Tax Foundation (1994). The National Finances: An Analysis of the Revenues and Expenditures of the Government of Canada. Toronto: Canada Tax Foundation

Comptroller General of Canada (1986). The Effects of the Access to Information Legislation on the Program Evaluation Function. Orawa: Program Evaluation Branch, Office of the Compuoller General of Canada.

De Koven, Harriet L. (1993). "Federal-Provincial Transfers: Which Way From Here?," Policy Options, Vol. 14, No. 10, pp. 45-48.

Dyck, Rand (1993). Canadian Politics: Critical Approaches. Scarborough: Nelson Publishers.

HaRTE, D. (1990). "Increasing Government Accountability: A Proposal that the Senate Assume Responsibility for Program Evaluation". Proceedings of the Senate Standing Committee on National Finance. 3 May, Appendix NF-23a.

Johnson, A. W. (1992). "Reflections on administrative reform in the Govemment of Canada, 1962-1991: A discussion paper," prepared for Office of the Auditor General, Ottawa.

Maltory, J. (1965). "The Five Faces of Canadian Federalism" in P. A. Crepeau and C. B. Macpherson (eds.). The Future of Canadian Federalism. Toronto: University of Toronto Press.

ONYSHKO, T. (1991). "Secret paper shows public defender system considered." The Lauyers Weekly (December 13, p. 9).

Poel, D. H., Aucoin, P., Conway, R. (1980). Nova Scotia Legal Aid: A Review and Proposal for Evaluation. Halifax: The Nova Scotia Legal Aid Commission and Federal Department of Justice.

Poel, D. H. (1983) The Nova Scotia Legal Aid Evaluation Report: Entering the "Third Generation". Halifax: The Nova Scotia Legal Aid Commission and the Federal Deparment of Justice.

PoEl, D. H. (1993). "Cooperation and Conflict in the Evaluation of Legal Aid Services in the Canadian Provinces," in J. Hudson and J. Roberts (editors), Evaluating Justice: Canadian Policies and Programs. Toronto: Thompson Educational Publishing, Inc., 1993.

Policy Options (1993). Fiscal Federalism: Debating Canada's Future (Vol. 14, No.10) Montreal: Institute for Research and Public Policy.

SEGSWORTH, R. V. (1990). Policy and Program Evaluation in the Government of Canada in R. C. Rist (ed.). Program Evaluation and the Management of Government. New Brunswick, N.J.: Transaction Publishers.
SEGSFORTH, R. V. (1992). Govemmental Activity Review in Ontario in J. Hudson, J. Mayne, and R. Thomlinson eds. Action-Oriented Evaluation. Toronto: Emerson and Wall.

SEGSwORTH, R. V. (1993). Public Access to Evaluation in Canada in R. Conner, J. Hudson, J. Mayne, and M. L. Bemelmans-Videc eds. Advancing Public Policy Evaluation. Amsterdam: North Holland.

SmileY, D. (1987). The Federal Condition in Canada. Toronto: McGraw-Hill Ryerson limimted.

Task Force on Program Review (198j). Introduction to the Process of Program Review. Orawa: Supply and Services.

Thomas, J. F. (1990). "Opening Statement," Proceedings of the Senate Standing Committee on National Finance, (25) 17 May.

TOUREMONDE, Jacques and Olaf REPER, editors (forthcoming). Inter-governmental evaluation. New Brunswick, N.J.: Transaction Publishers.

Treasury Board (1994). Treasury Board Manual on Review, Internal Audit and Evaluation. Ottawa: Treasury Board Secretariat.

WhEARE, K. C. (1946). Federal Government. London: Oxford University Press. RELACIÓN DE SIGLAS

ACOA Atlantic Canada Opportunities Agency (Agencia de Oportunidades del Canadá Atlántico.

BQ Bloc Québecois (federal Party) (Bloque de Quebec, partido federal).

CAP Canadá Assistance Plan (Plan de Ayuda Canadá).

EOP Employment Opportunities Program (Programa de Oportunidades de Empleo).

EPF Established Programs Fundings (Fondo para los Programas Establecidos).

DRDP Economic Regional Development Program (Programa para el desarnollo económico regional).

FEDNOR Federal Economic Development for Northern Ontario Region (Desarrollo económico federal para la región del Norte de Ontario).

NDP New Democratic Party (Nuevo Partido Democrático).

OLAP Ontario Legal Aid Plan (Plan de Asistencia Jurídica de Ontario).

PQ Parti Québecois (Partido de Quebec).

WED Western Economic Diversification Department (Departamento para la diversificación económica de Occidente). 


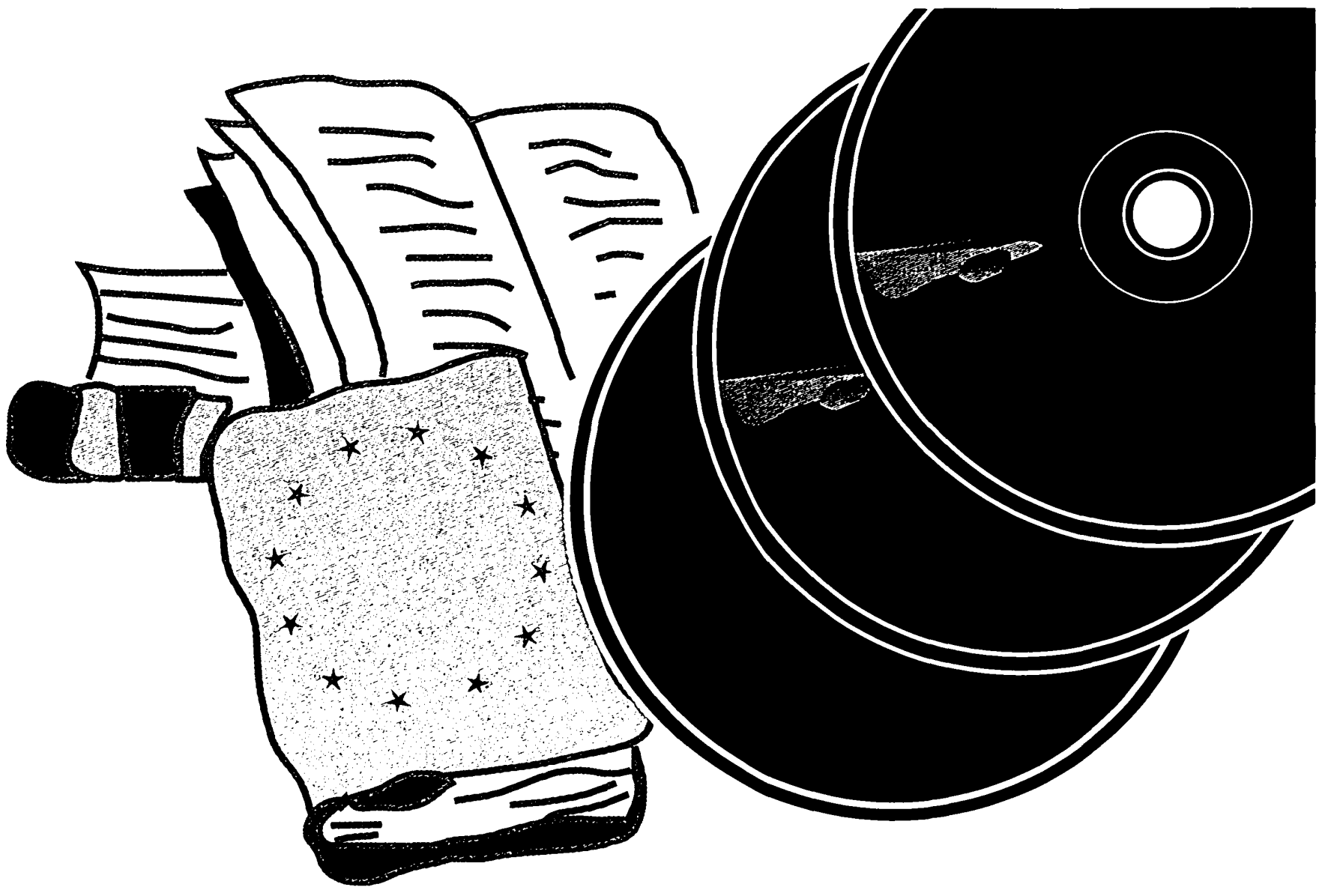

Lo último del BOE La legislación española, europea y todas las leyes sobre su Comunidad Autónoma, en CD-ROM.

Ahora a través de un CD-ROM, Disco óptico de gran rapidez y capacidad y que sólo necesita un PC y un lector para su 'consulta, $\mathrm{Vd}$. puede acceder a las nuevas bases de datos del BOE.

IBERLEX: Legislación Española y Europea, con disposiciones de carácter general desde 1968 hasta hoy.

MAP-LEXTER: Información juridica sobre administraciones territoriales

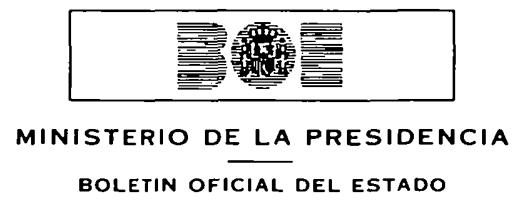

Infórmese en el (91) 5382222

$Y$ recuerde que también puede consultar esta información a través de los sistemas on-line y videotex. 